\title{
Repetitive control system: a new type servo system for periodic exogenous signals
}

$\operatorname{AUTHOR}(S):$

HARA, S; YAMAMOTO, Y; OMATA, T; NAKANO, M

\section{CITATION:}

HARA, S ... [et al]. Repetitive control system: a new type servo system for periodic

exogenous signals. IEEE TRANSACTIONS ON AUTOMATIC CONTROL 1988, 33(7): 659-668

\section{ISSUE DATE:}

1988-07

URL:

http://hdl.handle.net/2433/50291

\section{RIGHT:}

(c) 1988 IEEE. Personal use of this material is permitted. However, permission to reprint/republish this material for advertising or promotional purposes or for creating new collective works for resale or redistribution to servers or lists, or to reuse any copyrighted component of this work in other works must be obtained from the IEEE. 


\title{
Repetitive Control System: A New Type Servo System for Periodic Exogenous Signals
}

\author{
SHINJI HARA, MEMBER, IEEE, YUTAKA YAMAMOTO, MEMBER, IEEE, TOHRU OMATA, AND \\ MICHIO NAKANO, MEMBER, IEEE
}

\begin{abstract}
In this paper, a new control scheme called repetitive control is proposed, in which the controlled variables follow periodic reference commands. A high accuracy asymptotic tracking property is achieved by implementing a model that generates the periodic signals of period $L$ into the closed-loop system. Sufficient conditions for the stability of repetitive control systems and modified repetitive control systems are derived by applying the small gain theorem and the stability theorem for time-lag systems. Synthesis algorithms are presented both by the state-space approach and the factorization approach. In the former approach, the technique of the Kalman filter and perfect regulation is utilized, while coprime factorization over the matrix ring of proper stable rational functions and the solution of the Hankel norm approximation are used in the latter one.
\end{abstract}

\section{INTRODUCTION}

$\mathrm{NE}$ of the basic requirements in control systems is that they have the ability to regulate the controlled variables to reference commands without a steady-state error against unknown and unmeasurable disturbance inputs. Control systems with this property are called servo systems. In servo system design, the internal model principle proposed by Francis and Wonham [1] plays an important role. The internal model principle means that the controlled output tracks a class of reference commands without a steady-state error if the generator for the references is included in the stable closed-loop system. For example, no steadystate error occurs for step reference commands in a type 1 stable feedback system which has an integrator $1 / s$ (i.e., the generator of step functions) in the loop.

In practice, we often encounter the situation where the reference commands to be tracked and/or disturbance inputs to be rejected are periodic signals of a fixed period $L$, e.g., repetitive commands or operations for mechanical systems such as industrial robots and NC machines or disturbances depending on the frequency of the power supply. Any periodic signal can be generated by a free system including a time-lag element corresponding to the period with an appropriate initial function. Although nonclassical, it is natural to expect, in view of the internal model principle, that the desired asymptotic tracking for all periodic exogenous signals of period $L$ may be achieved by implementing a model which generates such periodic signals. It has been first pointed out by Inoue et al. [2], [3] that this assertion is true for linear SISO systems and it has been verified that this scheme is useful for some practical applications [2]-[5]. This scheme is called repetitive control and is useful for periodic reference commands and disturbance inputs. However, the stability conditions and the synthesis algorithms given there have

Manuscript received April 28, 1987; revised October 19, 1987. This paper is based on a prior submission of July 1, 1986. Paper recommended by Past Associate Editor, J. B. Pearson.

$\mathbf{S}$. Hara and M. Nakano are with the Department of Control Engineering, Tokyo Institute of Technology, Oh-Okayama, Meguro-Ku, Japan

Y. Yamamoto is with the Department of Applied Systems Science, Kyoto University, Yoshida, Sakyo-ku, Japan.

T. Omata is with the Electrotechnical Laboratory, Tsukuba, Japan. IEEE Log Number 8821358. not been generalized theoretically to multivariable systems. The present paper is, therefore, devoted to the stability analysis and control system design in multivariable repetitive control systems.

Repetitive control is regarded as a simple learning control because the control input is calculated using the information of the error signal in the preceding periods. An analogous scheme called betterment process has been developed for mechanical systems, and high accuracy control is also achieved by iteration of the control action [6]-[10]. In their methods, the reference command is corrected by means of the error signal obtained in the preceding trial. The difference between these two schemes is as follows. In the proposed repetitive control, the repetitive operation is done continuously, i.e., the initial state at the start of each period is equal to the final state of the preceding period. Therefore, the closed-loop system is a retarded or neutral type time-lag system [11] and it is not easy to stabilize the system. On the other hand, the same initial condition is assumed in every trial in the betterment process, hence, the iterative action is discrete and it is enough to assure not only the stability but the convergence of the error.

This paper is organized as follows. We introduce the repetitive control system and state the basic principle in Section II. A stability condition is derived by applying the small gain theorem to an equivalent system. In Section III, the modified repetitive control system with a low-pass filter in the repetitive control loop is introduced to relax the stability condition, which is derived by a stability theorem for systems with the time delays. Synthesis algorithms are presented both by the state-space approach and the factorization approach in Section IV. In the former approach, the technique of the Kalman filter plus perfect regulation [12] is utilized, while a coprime factorization over the matrix ring of proper stable rational functions [13] and the solution of the Hankel norm approximation [14] are used in the latter.

Notation used in this paper is as follows. $\mathscr{L}^{-1}[\cdot]$ means the inverse Laplace transform. A function $f(t)$ is called an $L_{2}$ function denoted by $f(t) \in L_{2}$ if $\int_{0}^{\infty} f(t) f(t) d t<\infty$. A rational function or matrix is said to be stable if it is analytic in the closed right-half complex plane, proper if it is finite at $s=\infty$, and strictly proper if it is zero at $s=\infty$. The sets of all proper, strictly proper, and proper stable rational $p \times m$ matrices are denoted by $\boldsymbol{R}_{p}^{p \times m}$, $\boldsymbol{R}_{s p m}^{p \times m}$, and $\boldsymbol{R}_{-}^{p \times m}$, respectively. When no confusion arises, we drop the superscript " $p \times m$ " for simplicity. The infinity norm of $G(s) \in \boldsymbol{R}_{-}$is defined by $\|G\|_{\infty} \triangleq \sup _{\omega} \bar{\sigma}[G(j \omega)]$, where $\bar{\sigma}[\cdot]$ denotes the largest singular value of the matrix. A rational matrix $G(s) \in \boldsymbol{R}_{-}(s)$ is said to be inner (respectively, co-inner) if $G^{*}(j \omega) G(j \omega)=I$ (respectively, $G(j \omega) G^{*}(j \omega)=I$ ) for all $\omega$, where * denotes the conjugate transpose of the matrix; $G(s)$ is said to be outer (respectively, co-outer) if $G(s)$ has full row (respectively, column) rank at all $\operatorname{Re} s>0$.

\section{Principle of Repetitive Control}

Any periodic signal with period $L$ can be generated by the free time-delay system shown in Fig. 1(a) with an appropriate initial function. The system has infinitely many poles on the imaginary 


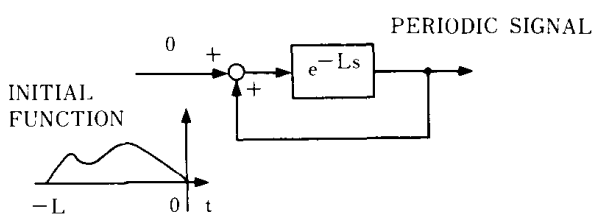

(a)

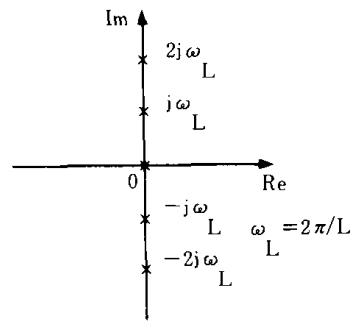

(b)

Fig. 1. Generator of periodic signal.

axis: $j k \omega_{L} ; k=0, \pm 1, \pm 2, \cdots$, where $\omega_{L}=2 \pi / L$ [see Fig. 1 (b)]. It is therefore expected from the internal model principle [1] that the asymptotic tracking property for exogenous periodic signals may be achieved by implementing the model exp $(-L s)$ $(1-\exp (-L s))$ into the closed-loop system. A controller including this model is said to be a repetitive controller and a system with such a controller is called a repetitive control system.

We now consider the repetitive control system with the model $a(s)+\exp (-L s) /(1-\exp (-L s))$ depicted in Fig. 2, where $R(s), Y_{p}(s)$, and $E(s)$ are the Laplace transforms of the reference command $r(t)$, controlled output $y_{p}(t)$, and error signal $e(t)$, respectively. $G(s) \in \boldsymbol{R}_{p}^{p \times p}$ denotes the transfer matrix for the compensated plant, and $a(s)$ is an appropriate proper stable rational function. Then the following relations hold:

$$
\begin{gathered}
E(s)=R(s)-Y_{p}(s), \\
Y_{p}(s)=G(s) V(s)+\bar{Y}(s), \\
V(s)=a(s) E(s)+W(s), \\
W(s)=\exp (-L s)[W(s)+E(s)]+\bar{W}(s)
\end{gathered}
$$

where $\bar{Y}(s)$ and $\bar{W}(s)$ are the Laplace transforms of the responses for initial conditions of $G(s)$ and $\exp (-L s) I$, respectively.

In previous work on SISO systems [1]-[3], the stability problem of repetitive control has been studied by transforming the system to an equivalent one as follows. From (2.1)-(2.4), we obtain $(I+a G) E=\exp (-L s)[I+(a-1) G] E+D_{e}$, i.e.,

$$
E=\exp (-L s)(I+a G)^{-1}[I+(a-1) G] E+(I+a G)^{-1} D_{e}
$$

where

$$
D_{e}=(1-\exp (-L s))(R-\bar{Y})-G \bar{W} .
$$

These equations lead to an equivalent system shown in Fig. 3. Let us discuss the error convergence condition or stability condition for the repetitive control system by considering the BIBO stability for the equivalent system described by $(2.5)$ with an aid of the small gain theorem [15]. Suppose that all the elements of $r(t)$ are bounded and continuous periodic signals with period $L$. Denote this by $r(t) \in P(L)$. This assumption yields that $r_{o}(t)$ is an $L_{2}$ function, where

$$
r_{o}(t) \triangleq \mathcal{L}^{-1}[(1-\exp (-L s)) R(s)]
$$

because $r_{o}(t)=r(t)$ is bounded for $0 \leqq t \leqq L$ and $r_{o}(t)=0$ for $t$

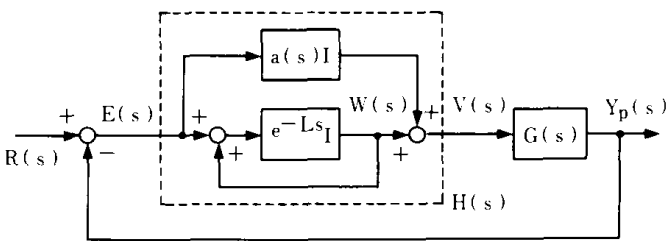

Fig. 2. Repetitive control system.

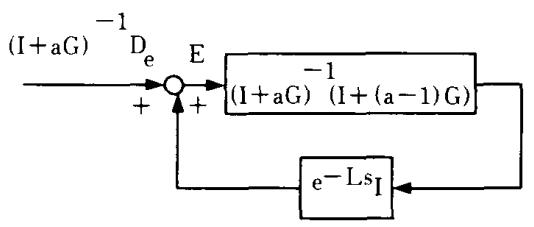

Fig. 3. A system equivalent to Fig. 2 .

$\geqq L$. This fact, together with (2.5), implies that the equivalent exogenous input $\mathcal{L}^{-1}\left[(I+a G)^{-1} D_{e}\right]$ is an $L_{2}$ function under the assumption of the asymptotic stability of $(I+a G)^{-1} G$. Under this setting, we have the following proposition.

Proposition 1: In the repetitive control system shown in Fig. 2,

$$
\begin{aligned}
& \text { 1) }[I+a(s) G(s)]^{-1} G(s) \in R_{-}, \\
& \text {2) }\left\|(I+a G)^{-1}[I+(a-1) G]\right\|_{\infty}<1
\end{aligned}
$$

then

$$
e(t)=\mathscr{L}^{-1}[E(s)] \in L_{2}
$$

for $r(t) \in P(L)$

Proof: First observe that $[I+a(s) G(s)]^{-1}$ belongs to $\boldsymbol{R}_{-}$in view of $(2.8)$ and $[I+a(s) G(s)]^{-1}=I-a(s)[I+$ $G(s) a(s)]^{-1} G(s)$, since $a(s)$ is a stable rational function. Hence, $[I+a(s) G(s)]^{-1}[I+(a(s)-1) G(s)]$ belongs to $\boldsymbol{R}_{-}$. Since the induced $L_{2}$ norm of $\mathfrak{L}^{-1}[G(s)]$ is less than or equal to $\|G\|_{\infty}$ and $|\exp (-j \omega L)|=1$ for all $\omega$, the result follows from the small gain theorem [15].

This sufficient condition for stability is very close to a necessary condition in a high-frequency range, since the phase shift caused by the delay exp $(-j \omega L)$ in the equivalent system can take any value at high frequencies. Observing that $(I+a G)^{-1}[I$ $+(a-1) G]=[I+(a-1) G](I+a G)^{-1},(2.9)$ can be rewritten as

$$
\begin{aligned}
& {\left[I+a^{*}(j \omega) G^{*}(j \omega)\right]^{-1}\left[I+\left(a^{*}(j \omega)-1\right) G^{*}(j \omega)\right]} \\
& \cdot[I+(a(j \omega)-1) G(j \omega)][I+a(j \omega) G(j \omega)]^{-1} \leqq \epsilon I<I ; \forall \omega
\end{aligned}
$$

for some real number $\epsilon<1$, or equivalently

$\left[I+\left(a^{*}(j \omega)-1\right) G^{*}(j \omega)\right][I+(a(j \omega)-1) G(j \omega)]$

$$
\leqq \epsilon\left[I+a^{*}(j \omega) G^{*}(j \omega)\right][I+a(j \omega) G(j \omega)] ; \forall \omega .
$$

The last inequality implies

$$
\begin{aligned}
G(j \omega)+G^{*}(j \omega)+\left(a(j \omega)+a^{*}(j \omega)-1\right) G^{*}(j \omega) G(j \omega) \\
\geqq(1-\epsilon)\left(I+a^{*}(j \omega) G^{*}(j \omega)\right)(I+a(j \omega) G(j \omega))>0 ; \forall \omega
\end{aligned}
$$

and then we have

$$
\begin{aligned}
\inf _{\omega} \lambda_{\min }\left[G^{*}(j \omega)+G(j \omega)\right. & +\left(a^{*}(j \omega)\right. \\
& \left.+a(j \omega)-1) G^{*}(j \omega) G(j \omega)\right]>0
\end{aligned}
$$

where $\lambda_{\min }[A]$ denotes the minimum eigenvalue of a Hermitian 
matrix $A$. Since (2.9) is also equivalent to

$[I+a(j \omega) G(j \omega)]^{-1}[I+(a(j \omega)-1) G(j \omega)]$

$\cdot\left[I+\left(a^{*}(j \omega)-1\right) G^{*}(j \omega)\right]\left[I+a^{*}(j \omega) G^{*}(j \omega)\right]^{-1} \leqq \epsilon I<I ; \forall \omega$ for some real number $\epsilon<1$, we obtain

$$
\begin{aligned}
\inf _{\omega} \lambda_{\min }\left[G(j \omega)+G^{*}(j \omega)\right. & +(a(j \omega) \\
\left.\left.+a^{*}(j \omega)-1\right) G(j \omega) G^{*}(j \omega)\right] & >0 .
\end{aligned}
$$

These inequalities (2.11a) and (2.11b) are closely related to the optimality condition of the optimal regulator or the Kalman filter in the case of $a(s)$ being a constant, especially when $a(s)=1$. For SISO systems (2.11) requires that the Nyquist plot of $G(s)$ remains inside the shadowed domain in Fig. 4, i.e., the locus $G(j \omega)$ should lie inside the unit circle centered at $1+j 0$, or outside the unit circle centered at $-1+j 0$, according to $a(s)=0$ or $a(s)=$ 1. We also note that for $a(s)=1 / 2$, the loci should remain in the open right-half complex plane, in other words, $G(s)$ is to be strictly passive [15].

Proposition 1 summarizes how repetitive control has been studied in the literature. However, there are two unsatisfactory points in Proposition 1 . One is that it guarantees only the $L_{2}$-BIBO stability. Since we are interested in whether or not the error $e(t)$ actually tends to zero, the conclusion $e(t) \in L_{2}$ may be a little too weak for the purpose of the servo problem. This difficulty will be overcome by adopting a more powerful stability criterion in the next section.

Another weakness is that condition (2.9) can be strictly satisfied only for systems with a direct path, i.e., systems with relative degree zero, otherwise, $G(j \omega) \rightarrow 0$ as $\omega \rightarrow \infty$. Actually, we can prove that it is impossible to construct a repetitive controller, which exponentially stabilizes the repetitive control system, for strictly proper plants.

Proposition 2: Let $G(s)$ be a strictly proper transfer function matrix. Then the repetitive control system Fig. 2 cannot be exponentially stable.

Proof: See Appendix A.

The nonexistence of a repetitive controller (in the strict sense) for a strictly proper plant is not surprising in the following sense. In the servo theory, it is well known that output regulation is possible only when plant zeros do not cancel the poles of the reference signal generator. Extending this principle to the present situation (although it is nonclassical), we see that this principle is not satisfied for a strictly proper plant $G(s)$, for $G(s)$ has infinity as its zero, whereas the generator of the periodic signal has a pole of arbitrarily high frequency. To put it differently, if $G(s)$ is strictly proper, then it integrates the input at least once, and hence the output will be smoothed out to some extent, thereby making it impossible to track a signal with an infinitely sharp edge, i.e., a signal containing arbitrarily high-frequency modes.

This is unfortunate, but not entirely irreconcilable since this is caused by the apparently unrealistic demand of tracking any periodic signal, which contains arbitrarily high-frequency modes. It is therefore natural to expect that the stability condition can be relaxed by reducing the loop-gain of the repetitive compensator in a higher frequency range. This leads to the idea of a modified repetitive control system in which we replace the delay element $\exp (-L s)$ by $q(s) \exp (-L s)$ for a suitable proper stable rational $q(s)$ such that $|q(j \omega)|<1$ for all $\omega$ larger than a chosen cutoff frequency $\omega_{c}$. This low-pass filter may be realized by a simple first-order system $q(s)=1 /(1+T s), T>0$, or $q(s)=(1+$ $\left.T_{2} s\right) /\left(1+T_{1} s\right), T_{1}>T_{2}>0$, for example. The stability analysis of this modified repetitive control system is the theme of the next section.

\section{Modified Repetitive Control System}

We discuss the stability condition and properties of the modified repetitive control system depicted in Fig. 5, where a

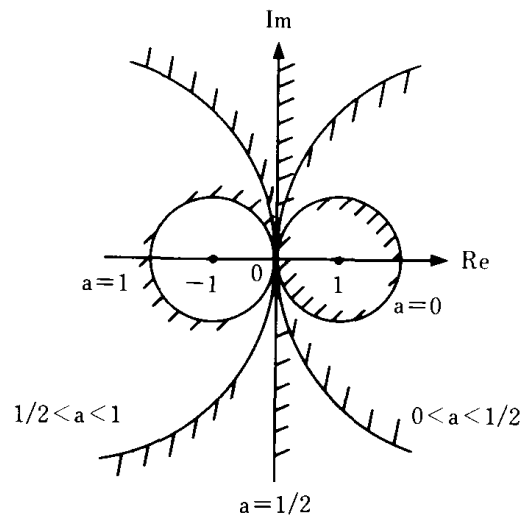

Fig. 4. Stability circles for repetitive control system.

low-pass filter $q(s)$ is an appropriate proper stable rational function (note that $a(s)$ need not be strictly proper). As in the discussion on the repetitive control system in the previous section

$E=\exp (-L s) q[I+a G]^{-1}[I+(a-1) G] E+[I+a G]^{-1} D_{m}$ (3.1)

where

$$
D_{m}=(1-\exp (-L s) q)(R-\bar{Y})-G \bar{W} .
$$

Equation (3.1) represents a system equivalent to the modified repetitive control system, whose block diagram is shown in Fig. 6 . This equivalent system turns out to be a retarded or neutral time-lag system according to $q(\infty)=0$ or $q(\infty) \neq 0$. We now obtain the following stability condition by using the general stability result on time-lag systems [11], [16]. [17].

Theorem $1:$ In the modified repetitive control system shown in Fig. 5 with $G(s) \in \boldsymbol{R}_{p}^{p \times p}, a(s) \in \boldsymbol{R}_{-}^{1 \times 1}$, and $q(s) \in \boldsymbol{R}_{-}^{1 \times 1}$, if

1) $[I+a(s) G(s)]^{-1} G(s) \in \boldsymbol{R}_{-}$,

2) $\|Q\|_{\infty}<1$

where

$$
Q \triangleq q(I+a G)^{-1}[I+(a-1) G]
$$

then the system with minimal realization is exponentially stable. Moreover, the error $e(t)$ is bounded for any command $r(t) \in$ $P(L)$ and if $q(s)=1$, i.e., in the repetitive control case

$$
\lim _{t \rightarrow \infty} e(t)=0
$$

holds.

Proof: Observe that as in the proof of Proposition 1, $[I+$ $a G]^{-1}$ belongs to $\boldsymbol{R}_{-}$and hence $Q \in \boldsymbol{R}_{-}$. Let

$$
\begin{aligned}
& \dot{x}(t)=A x(t)+B u(t) \\
& y(t)=C x(t)+D u(t)
\end{aligned}
$$

be a minimal realization of $Q(s)$, where $x(t) \in \mathbb{R}^{n}, u(t) \in \mathbb{R}^{p}$, and $y(t) \in R^{p}$. In view of Fig. 6 , the system (3.1) with $D_{m}=0$ is described by the following well-known functional differential equation model [18]:

$$
\begin{gathered}
\frac{d}{d t}\left[\begin{array}{l}
x_{t} \\
z_{t}
\end{array}\right]=\left[\begin{array}{c}
A x_{t}+B z_{t}(-L) \\
\frac{\partial}{\partial \theta} z_{t}(\theta)
\end{array}\right], \\
y(t)=z_{t}(0) .
\end{gathered}
$$

Here $x_{t}$ is the state of the integrator in $Q(s)$, and $z_{t}(\theta)$ is the state 


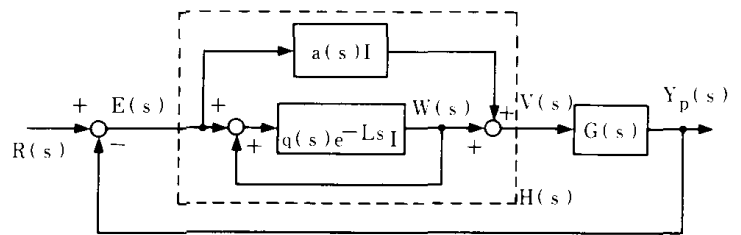

Fig. 5. Modified repetitive control system.

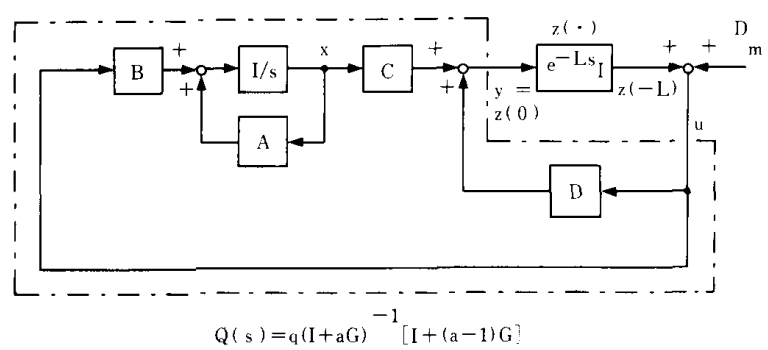

Fig. 6. A system equivalent to Fig. 5 .

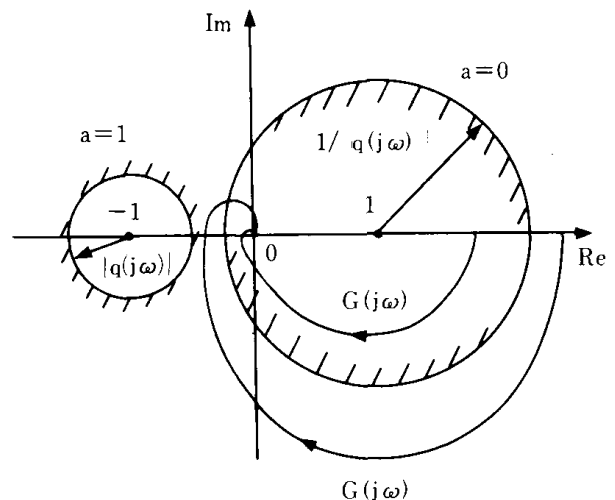

Fig. 7. Stability circles for modified repetitive control system

in the delay which is a function of $-L \leqq \theta \leqq 0$ at each $t$ (see Fig. 7). The pair $\left(x_{t}, z_{t}(\cdot)\right)$ is required to belong to the state-space $R^{n} \times\left(L_{2}[-L, 0]\right)^{p}$. Denote the operator on the right-hand side of (3.8a) by $A$. The domain $D(A)$ of $A$ is specified as

$D(A) \triangleq\left\{(x, z(\cdot)) \in \mathbb{R}^{n} \times\left(L_{2}[-L, 0]\right)^{p}\right.$

$$
\left.z(\cdot) \in W_{2}^{1}[-L, 0], z(0)=C x+D z(-L)\right\} .
$$

Here $W_{2}^{1}[-L, 0]$ is the first-order Sobolev space. The equation $z(0)=C x+D z(-L)$ in this definition specifies how $C x_{t}$ and the delayed feedback is connected with the input terminal $z_{t}(0)$ in the delay.

It is known [11], [16], [17] that this system is exponentially stable if

$$
\sup \{\operatorname{Re} \lambda ; \lambda \in \sigma(A)\}<0
$$

where $\sigma(A)$ is the spectrum of $A$. Let us compute $\sigma(A)$. Since it is known that $\sigma(A)$ consists only of eigenvalues [11], we have that $\lambda$ belongs to $\sigma(A)$ if and only if

$$
\begin{gathered}
\lambda x-A x-B z(-L)=0, \\
\lambda z-\frac{d}{d \theta} z(\theta)=0
\end{gathered}
$$

for a nonzero $(x, z) \in D(A)$, i.e., $z(0)=C x+D z(-L)$.
Equation (3.11b) implies that $z(\theta)=v \exp (\lambda \theta)$ with an appropriate $R^{p}$ vector $v$, so that $(3.11 \mathrm{a})$ and the condition $(x, z) \in$ $D(A)$ reduce to

$$
\left[\begin{array}{cc}
\lambda I-A & -B \exp (-\lambda L) \\
-C & I-D \exp (-\lambda L)
\end{array}\right]\left[\begin{array}{l}
x \\
v
\end{array}\right]=0
$$

Now (3.12) admits a nontrivial solution if and only if

$$
\begin{aligned}
0= & \operatorname{det}\left[\begin{array}{cc}
\lambda I-A & -B \exp (\lambda-L) \\
-C & I-D \exp (-\lambda L)
\end{array}\right] \\
= & \operatorname{det}(\lambda I-A) \operatorname{det}\left[(I-D \sigma)-C(\lambda I-A)^{-1} B \sigma\right] \\
& (\sigma=\exp (-\lambda L)) \\
= & \operatorname{det}(\lambda I-A) \operatorname{det}[I-Q(\lambda) \exp (-\lambda L)] .
\end{aligned}
$$

Therefore, condition $(3.10)$ reduces to

$$
\gamma \triangleq \sup \{\operatorname{Re} \lambda ; \operatorname{det}[I-Q(\lambda) \exp (-\lambda L)]=0\}<0
$$

under the hypothesis that $Q(s)$ is stable, i.e., $A$ is a stable matrix

We now show (3.14) under the condition of (3.4). If (3.4) holds, then there exists a positive number $\rho$ such that $\sigma[Q(\lambda)] \leqq \rho$ $<1$ holds for any $\operatorname{Re} \lambda \geq 0$ [13]. This means that, for every $\lambda$ with $\operatorname{Re} \lambda \geqq 0$, the magnitude of the eigenvalues of $Q(\lambda) \exp (-$ $\lambda L)$ is less than or equal to $\rho$, and hence $[I-Q(\lambda) \exp (-\lambda L)]$ has no eigenvalues at the origin. This yields that det $[I-Q(\lambda)$ $\exp (-\lambda L)] \neq 0$ for $\operatorname{Re} \lambda \geqq 0$ which implies $\gamma \leqq 0$. Consequently, it is enough to show $\gamma \neq 0$. Suppose that $\gamma=0$. Then there exists a sequence $\left\{\lambda_{i}=x_{i}+y_{i} ; x_{i}<0\right\}$ such that det $\left[I-Q\left(\lambda_{i}\right) \exp \left(-\lambda_{i} L\right)\right]=0$ and $x_{i} \rightarrow 0$ as $i \rightarrow \infty$. This means that $\exp \left(\lambda_{i} L\right)$ is an eigenvalue of $Q\left(\lambda_{i}\right)$. Since $\left|\exp \left(\lambda_{i} L\right)\right| \rightarrow 1$, it follows that

$$
\bar{\sigma}\left[Q\left(\lambda_{i}\right)\right] \geqq 1-\epsilon_{i} ; \epsilon_{i} \rightarrow 0 .
$$

Since the maximum singular value of $Q(\lambda)$ is continuous with respect to $\lambda$ in a neighborhood of the imaginary axis by the asymptotic stability of $Q(s),(3.15)$ yields that the supremum of the maximum singular value of $Q(j \psi)$ must be no less than 1 , i.e., $\|Q\|_{\infty} \geqq 1$. This contradicts our hypothesis (3.4), and hence we conclude $\gamma<0$.

The exponential stability of the closed-loop system and the boundedness of the equivalent external input $\mathscr{L}^{-1}\left[(I+a G)^{-1} D_{m}\right]$ leads to the boundedness of the error $e(t)$. In particular, if $q(s)=$ 1 then (3.6) holds, since the equivalent external input $\mathscr{L}^{-1}[(1-$ $\left.\exp (-L s))(I+a G)^{-1} R\right]$ goes to 0 as $t$ tends to $\infty$.

The result of Theorem 1 contains that of Proposition 1, because Theorem 1 is concerned with any $q(s) \in R_{-}$while $q(s)=1$ in Proposition 1. Further, Theorem 1 assures asymptotic stability or uniform convergence (3.6) while Proposition 1 guarantees only the $L_{2}$ input/output stability (2.10).

It is readily seen from condition (3.4) that the stability condition becomes milder as $|q(j \omega)|$ becomes close to 0 . As shown in Fig. 8 for SISO systems the Nyquist plot of $G(s)$ should lie inside respectively, outside) the circle in the complex plane of radius $1 /$ $|q(j \omega)|$ (respectively, $|q(j \omega)|)$ centered at $1+j 0$ (respectively, $-1+j 0)$. However, when we make $|q(j \omega)|$ closer to 0 for improving the stability, it deteriorates tracking as it is distant from 1 , because the desired poles $2 k \pi j / L$ for precise tracking are altered by $q(s)$. Since this tradeoff relationship between stability and tracking is frequency-dependent, it is desirable (and possible) to take the filter $q(s)$ in such a way that it is close (preferably equal) to 1 in a low-frequency range where tracking is important and that it is less than 1 (preferably close to 0 ) in the higher frequency range to improve on the stability condition. Since lowfrequency band is dominant in any reference signal, this will virtually satisfy any practical demands. Therefore, a typical 


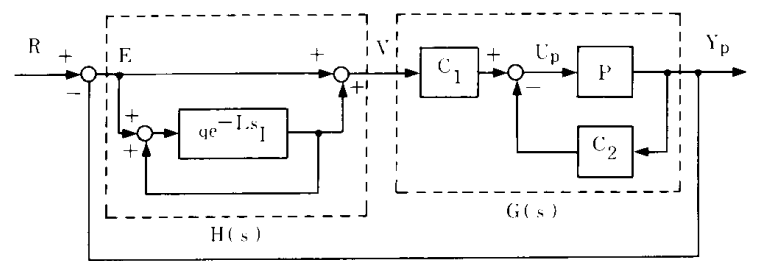

Fig. 8. Modified repetitive control system with two degrees of freedom.

desirable filter $q(s)$ should have the frequency characteristics:

$$
\begin{aligned}
& \text { 1) } q(j \omega) \sim 1 ;|\omega| \leqq \omega_{c} \\
& \text { 2) }|q(j \omega)| \leqq \rho<1 ;|\omega|>\omega_{c}
\end{aligned}
$$

for a suitable cutoff frequency $\omega_{c}$. We now estimate the tracking error in such a case where the reference signals have the frequency band lower than $\omega_{c}$.

Theorem 2: Take any bounded interval $\left[-\omega_{c}, \omega_{c}\right]$. Let $q_{i}(s) \rightarrow$ 1 uniformly on $\left[-\omega_{c}, \omega_{c}\right]$ such that $\left\|Q_{i}\right\|_{\infty} \leqq \rho<1$ independently of $i$, where $Q_{i}$ is given by (3.5) for $q=q_{i}$. Consider a modified repetitive control system (Fig. 5) with $q(s)=q_{i}(s)$. Suppose that the hypotheses of Theorem 1 are satisfied. Then for any reference signal $r(t) \in P(L)$ which contains the frequencies lower than $\omega_{c}$, the error $e_{i}(t)$ in the modified repetitive control system with $q=q_{i}$ satisfies

$$
\lim _{i \rightarrow \infty} \limsup _{m \rightarrow \infty}\left\|e_{i}(t)\right\|_{[m L,(m+1) L]}=0
$$

where $\|\cdot\|_{[m L,(m+1) L]}$ denotes the $L_{2}$-norm on $[m L,(m+1) L]$.

The same result holds also for uniform convergence.

Proof: Let $G^{i}(s)$ denote the transfer function matrix from $r$ to $e_{i}$ in Fig. 5. Let $N$ be the largest integer such that $\left|\omega_{N}\right|<\omega_{c}$, where $\omega_{k}=2 \pi k j / L$. Take any $0<\epsilon<1$. Since $G_{e r}^{i}(s)$ possesses zeros at $\left\{\lambda ; 1-q_{i}(\lambda) \exp (-\lambda L)=0\right\}$, and since $q_{i}(s) \rightarrow 1$ on $\left[-\omega_{c}, \omega_{c}\right]$, there exist zeros $\alpha_{k}^{i} \pm j \beta_{k}^{i}, k=0,1$, $\cdots N\left(\beta_{0}=0, \beta_{-k}^{i}=-\beta_{k}^{i}\right.$ for $\left.k \geqq 1\right)$ of $G_{e r}^{i}(s)$ such that

$$
\left|\alpha_{k}^{i}+j \beta_{k}^{i}-j \omega_{k}\right|<\epsilon ; k=0, \pm 1, \cdots, \pm N
$$

for all sufficiently large $i$. For notational simplicity, consider the tracking in the first channel and let $r(t)=\underline{e}_{1} \sin \left(\omega_{k} t\right)$, where $\underline{e}_{1}$ $\triangleq[1,0, \cdots, 0]^{T}$. Let us first consider the case $k \geqq 1$. Since $G_{e r}^{i}\left(\alpha_{0}\right)=0$ and $G_{e r}^{i}\left(\alpha_{k}^{i} \pm j \beta_{k}^{i}\right)=0$, we have $G_{e r}^{i}(s) \underline{e}_{1}=$ $\left[\left(s-\alpha_{k}^{i}\right)^{2}+\left(\beta_{k}^{i}\right)^{2}\right] \tilde{G}^{i}(s)$. Then

$$
\begin{aligned}
e_{i}(t)= & \mathcal{L}^{-1}\left[G_{e r}^{i}(s) \underline{e}_{1} /\left(s^{2}+\omega_{k}^{2}\right)\right] \\
= & \mathscr{L}^{-1}\left[\tilde{G}^{i}(s)\left\{\left(s-\alpha_{k}^{i}\right)^{2}+\left(\beta_{k}^{i}\right)^{2}\right\} /\left(s^{2}+\omega_{k}^{2}\right)\right] \\
= & \mathcal{L}^{-1}\left[\tilde{G}^{i}(s)\right]^{*}\left[\delta(t)+\left\{\left(\left(\alpha_{k}^{i}\right)^{2}+\left(\beta_{k}^{i}\right)^{2}\right.\right.\right. \\
& \left.\left.\left.-\omega_{k}^{2}\right) / \omega_{k}\right\} \sin \left(\omega_{k} t\right)-2 \alpha_{k}^{i} \cos \left(\omega_{k} t\right)\right]
\end{aligned}
$$

where $\delta(t)$ denotes the Dirac delta function. Since $G^{i}(s)$ is exponentially stable, $\tilde{G}^{i}(s)$ is also exponentially stable. Furthermore, since

$$
\begin{aligned}
& \left|\left(\alpha_{k}^{i}\right)^{2}+\left(\beta_{k}^{i}\right)^{2}-\omega_{k}^{2}\right| /\left|\omega_{k}\right| \\
& \quad \leqq\left\|\alpha_{k}^{i}+j \beta_{k}^{i}|-| j \omega_{k}\right\| \cdot\left\|\alpha_{k}^{i}+j \beta_{k}^{i}|+| j \omega_{k}\right\| /\left|\omega_{k}\right| \\
& \quad \leqq\left|\alpha_{k}^{i}+j \beta_{k}^{i}-j \omega_{k}\right| \cdot\left\|\alpha_{k}^{i}+j \beta_{k}^{i}|+| j \omega_{k}\right\| /\left|\omega_{k}\right| \\
& \quad \leqq \epsilon\left(2\left|\omega_{k}\right|+\epsilon\right) /\left|\omega_{k}\right|
\end{aligned}
$$

by (3.18), and since $\left|\omega_{k}\right| \geqq 2 \pi / L$, it easily follows that the $L_{2}$ norm of $e_{i}(t)$ on any period $[m L,(m+1) L]$ is bounded by $C_{0} \epsilon\left\|\sin \left(\omega_{k} t\right)\right\|_{[0, L]}+\eta_{0}^{i}(t)$, where $C_{0}$ is a constant depending on $\tilde{G}^{i}$, and $\eta_{0}^{i}(t)$ is the term representing $\mathscr{L}^{-1}\left[\tilde{G}^{i}(s)\right] * \delta(t)$ and the effect by the initial value which goes to 0 as $t \rightarrow \infty$. The case of $k$
$=0$ can be estimated similarly by noting $G_{e r}^{i}(s) \underline{e}_{1}\left[s+\alpha_{0}^{i}\right] \tilde{G}^{i}(s)$ in this case.

Now let $r(t)=\sum_{k} a_{k} \sin \left(\omega_{k} t\right)$. Since $\left\{\sin \left(\omega_{k} t\right)\right\}$ and $\left\{\cos \left(\omega_{k} t\right)\right\}$ are mutually orthogonal, we have

$$
\left\|e_{i}(t)\right\|_{[m L,(m+1) L]} \leqq C \epsilon\|r(t)\|_{[0, L]}+\eta^{i}(t)
$$

for a suitable constant $C$ depending on $G^{i}(s)$ and $N$, and $\eta^{i}(t) \rightarrow$ $0(t \rightarrow \infty)$. In view of the uniform bound $\left\|Q_{i}\right\| \leqq \rho<1$, it follows from Hale [11] and the proof of Theorem 1 that $G_{e r}^{i}(s)$, and hence $\tilde{G}^{i}(s)$, is exponentially stable uniformly in $i$. Therefore, the estimate (3.19) is independent of $i$, so that the right-hand side of (3.19) tends to 0 as $i \rightarrow \infty$. This proves (3.17).

Since the same stability result holds when we take the space of continuous functions as the state-space [11], and since $r(t)$ is continuous in the above, the error converges to zero also in the sense of uniform convergence.

\section{Synthesis of Repetitive Control Systems}

In this section, we consider the synthesis of modified repetitive control systems both by the state-space approach and by the factorization approach. For simplicity, we investigate only the case $a(s)=1$. However, a similar discussion can be carried out for the general case [19].

The control system investigated here is depicted in Fig. 8, where $P(s) \in \boldsymbol{R}_{s p}^{p \times m}$ denotes the strictly proper plant to be controlled and the controller consists of a cascade compensator $C_{1}(s) \in \boldsymbol{R}_{p}^{m \times p}$, a feedback compensator $C_{2}(s) \in \boldsymbol{R}_{p}^{m \times p}$, and a low-pass filter $q(s) \in \boldsymbol{R}^{1 \times 1}$. In this figure, the compensated plant $G(s)$ and the repetitive controller $H(s)$ are expressed as

$$
G(s)=\left(I+P(s) C_{2}(s)\right)^{-1} P(s) C_{1}(s)
$$

and

$$
\begin{aligned}
H(s) & =[1+\exp (-L s) q(s) /(1-\exp (-L s) q(s))] I_{p} \\
& =[1 /(1-\exp (-L s) q(s))] I_{p},
\end{aligned}
$$

respectively. The two-degree-of-freedom compensator $C(s)=$ $\left[C_{1}(s), C_{2}(s)\right]$ specifies the characteristics of the conventional feedback system without a repetitive action. The single freedom case, i.e., $C_{2}(s)=0$ will be also studied as a special case. The low-pass filter $q(s)$ governs both stability and steady-state characteristics. The stability condition of this system is thus stated as follows.

Corollary $1:$ In the modified repetitive control system shown in Fig. 8 with $P(s) \in \boldsymbol{R}_{s p}^{p \times m}, C_{i}(s) \in \boldsymbol{R}_{p}^{m \times p} ; i=1,2$, and $q(s) \in$ $R_{-}^{1 \times 1}$, if

$$
\text { 1) }[I+G(s)]^{-1} G(s) \in \boldsymbol{R}_{-}
$$

with no unstable pole-zero cancellation between $P(s)$ and $C(s)=$ $\left[C_{1}(s), C_{2}(s)\right]$, and

$$
\text { 2) }\left\|q(I+G)^{-1}\right\|_{\infty}<1
$$

hold, then the system with minimal realization is exponentially stable.

Synthesis of modified repetitive control systems satisfying the stability condition in Corollary 1 will be investigated both by the state-space approach and by the factorization approach in the following sections.

\section{A. State-Space Approach}

We propose a synthesis algorithm of modified repetitive control systems with $G(s)=P(s) C_{1}(s)$ (i.e., $C_{2}(s)=0$ ) in Fig. 8, for minimum-phase plants, i.e., systems without unstable zeros, by the state-space approach. A method of the Kalman filter with perfect regulation can be used in this algorithm because the 
stability condition is closely related to the optimality condition of the Kalman filter or of the optimal regulator as stated in Section II.

[A Synthesis Algorithm by the State-Space Approach]:

Step 1: Find a minimal realization $\left(A_{p}, B_{p}, C_{p}\right)$ of the given plant $P(s)$ :

$$
P(s)=C_{p}\left(s I-A_{p}\right)^{-1} B_{p} .
$$

Step 2: Construct a cascade compensator $C_{1}(s)$ whose configuration is shown in Fig. 9, by calculating the gains $F$ and $K$ as follows:

1) $F=\Sigma C_{p}^{T}$,

where $\Sigma$ is a positive definite solution of

$A_{p} \Sigma+\Sigma A_{p}^{T}+\Phi-\Sigma C_{p}^{T} C_{p} \Sigma=0$

with $\left(A_{p}, \Phi^{1 / 2}\right)$ being a controllable pair.

2) $K=K_{\rho}(\rho \rightarrow \infty)$,

where $K_{\rho}$ is a gain of perfect regulation [12].

Step 3: Choose an appropriate $q(s)$ so that the condition (4.4) holds and the system has the desired band-pass frequency characteristics.

The following fact shows that the designed control system satisfies the stability condition in Theorem 1 with $q(s)=1$ asymptotically as $\rho$ goes to infinity except at $\omega=\infty$.

If $C_{1}(s)$ is constructed as shown in Fig. 9, the transfer matrix of the compensated plant $G(s)\left(Y_{p}(s)=G(s) V(s)\right)$ is represented by

$$
\begin{aligned}
G(s)=\left[C_{p}\left(s I-A_{p}\right)^{-1}-C_{p}\left(s I-A_{p}+B_{p} K\right)^{-1}\right] \\
\cdot\left[I+F C_{p}\left(s I-A_{p}+B_{p} K\right)^{-1}\right]^{-1} F .
\end{aligned}
$$

Since the relation

$$
\lim _{\rho \rightarrow \infty} C_{p}\left(s I-A_{p}+B_{p} K_{\rho}\right)^{-1}=0
$$

holds for the gain $K=K_{\rho}$ of perfect regulation [12], substituting (4.10) into (4.9) yields

$$
G_{\infty}(s) \triangleq \lim _{\rho \rightarrow \infty} G(s)=C_{p}\left(s I-A_{p}\right)^{-1} F .
$$

Furthermore, it leads to the following circle condition:

$$
\left(I+G_{\infty}(j \omega)\right)\left(I+G_{\infty}(j \omega)\right)^{*}>I ; \forall \omega
$$

because $F$ is the gain of Kalman filter (4.6). Condition (2.11b), therefore, holds asymptotically as $\rho \rightarrow \infty$ except at $\omega=\infty$. The separation theorem guarantees condition 1) in Corollary 1 .

This implies that the modified repetitive control system with any tracking frequency band can be designed by setting the filter $q(s)$ as $|q(j \omega)| \leqq 1$ and $|q(\infty)|<1$ and $\rho \rightarrow \infty$ in the above algorithm

Example 1: Consider a SISO plant described by

$$
P(s)=1 /\left(s^{3}+2 s^{2}+2 s+1\right) .
$$

Step 1: Find the controllable canonical form $\left(A_{p}, B_{p}, C_{p}\right)$

$$
A_{p}=\left[\begin{array}{rrr}
0 & 1 & 0 \\
0 & 0 & 1 \\
-1 & -2 & -2
\end{array}\right], B_{p}=\left[\begin{array}{l}
0 \\
0 \\
1
\end{array}\right], C_{p}=\left[\begin{array}{lll}
1 & 0 & 0
\end{array}\right]
$$

as a minimal realization of $P(s)$.

Step 2: Let $\Phi=\operatorname{diag}(0,0,10)$ and calculate the gain of the Kalman filter $F$ by solving (4.7) and (4.6). The gain $K_{\rho}$ is determined by using the method of optimal control with quadratic

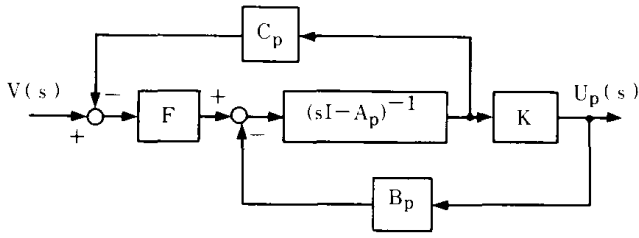

Fig. 9. Configuration of compensator $C_{1}(s)$.

performance index $J_{\rho}=\int_{0}^{\infty}\left(x^{T} Q_{\rho} x+u^{T} R u\right) d t$, where $Q_{\rho}=$ $\operatorname{diag}(\rho, 0,0)$ and $R=1$.

Fig. 10 indicates the loci of $|1+G(j \omega)|$ for $\rho=0\left(C_{1}(s)=\right.$ $I), \rho=10^{3}$ and $\rho=10^{5}$, where the gains $K_{\rho}$ for $\rho=10^{3}$ and $\rho$ $=10^{5}$ are $[99.0,41.1,7.28]^{T}$ and $[315.2,90.8,11.6]^{T}$, respectively. It is seen by this figure that the modified repetitive control systems with $q(s)=1 /(1+s)$ are stable for $\rho=10^{3}$ and $\rho=10^{5}$. On the other hand, the sufficient condition of stability (4.4) is not satisfied for $C_{1}(s)=I$, and the system is in fact unstable as illustrated in Fig. 11. We emphasize that very small steady-state errors occur in the modified repetitive control systems [see Fig. 12(b) and (c)] compared to those in the conventional feedback system, i.e., in the case of $q(s)=0$ [see Fig. 12(a)]. It is also verified from Fig. 12 that the steady-state error for $q(s)=1 /(1+0.56 s)$ is less than that for $q(s)=1 /(1$ $+s)$; in other words $q(s)$ has the wider frequency band to be tracked and has the smaller steady-state error as stated in the previous section.

\section{B. Factorization Approach}

In this section the classes of $C_{i}(s) ; i=1,2$ and $q(s)$ which satisfy the stability condition of Theorem 2 with $G(s)=[I+$ $\left.P(s) C_{2}(s)\right]^{-1} P(s) C_{1}(s)$ are clarified by using coprime factorization of the plant $P(s)$ over the ring of proper stable rational matrices [13].

Let

$$
P(s)=N(s) D(s)^{-1}, P(s)=\tilde{D}^{-1}(s) \tilde{N}(s)
$$

be right and left coprime factorizations, respectively. Suppose that the corresponding Bezout identities satisfy $U(s) V(s)=V(s) U(s)$ $=I$, where

$$
U(s)=\left[\begin{array}{rr}
Y(s) & X(s) \\
-\tilde{N}(s) & \tilde{D}(s)
\end{array}\right], V(s)=\left[\begin{array}{rr}
D(s) & -\tilde{X}(s) \\
N(s) & \tilde{Y}(s)
\end{array}\right] .
$$

Under these preliminaries any $C(s)=\left[C_{1}(s), C_{2}(s)\right]$ satisfying condition 1) in Corollary 1 can be written as

$$
C_{1}=\left(Y-K_{2} \tilde{N}\right)^{-1}\left(X+K_{1}\right), C_{2}=\left(Y-K_{2} \tilde{N}\right)^{-1}\left(-K_{1}+K_{2} \tilde{D}\right)
$$

with an appropriate $K_{i}(s) \in R_{-}^{m \times p} ; i=1,2$. (See Appendix B for a brief derivation; more precise and general investigation is in [20].) $K_{1}(s)$ and $K_{2}(s)$ are free parameters to be determined in the design. Using the Bezout equations $U(s) V(s)=V(s) U(s)=I$ and (4.15), we have

$$
\begin{aligned}
G= & P\left(I+C_{2} P\right)^{-1} C_{1} \\
= & N D^{-1}\left[I+\left(Y-K_{2} \tilde{N}\right)^{-1}\left(-K_{1}+K_{2} \tilde{D}\right)\right. \\
& \left.\cdot N D^{-1}\right]^{-1}\left(Y-K_{2} \tilde{N}\right)^{-1}\left(X+K_{1}\right) \\
= & N\left[\left(Y-K_{2} \tilde{N}\right) D+\left(-K_{1}+K_{2} \tilde{D}\right) N\right]^{-1}\left(X+K_{1}\right) \\
= & N\left[Y D-K_{1} N\right]^{-1}\left(X+K_{1}\right) \\
= & N\left[I-\left(X+K_{1}\right) N\right]^{-1}\left(X+K_{1}\right) \\
= & {\left[I-N\left(X+K_{1}\right)\right]^{-1} N\left(X+K_{1}\right) . }
\end{aligned}
$$




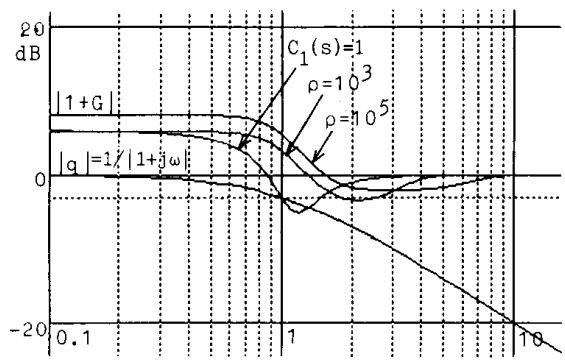

Fig. 10. Bode diagrams of $|1+G(j \omega)|$.
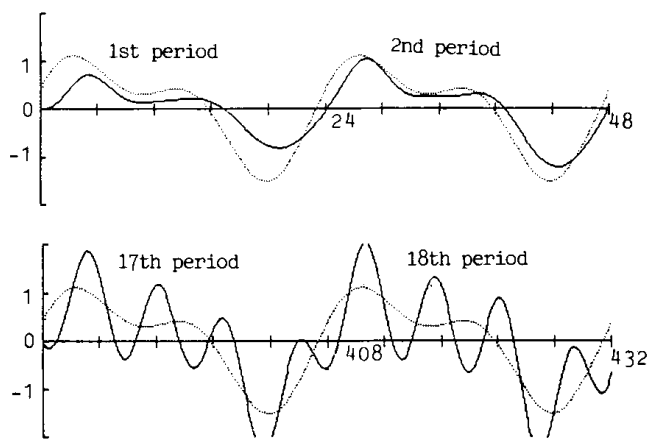

- output $y(t) \quad \ldots$ reference $r(t)$

Fig. 11. Response for the example: $\rho=0, T=1.0$.

(a)

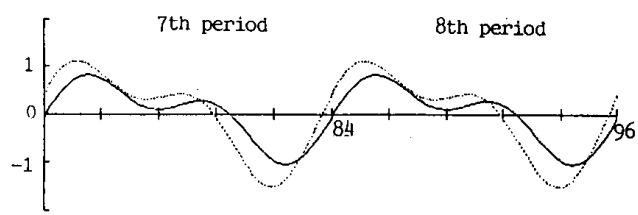

(b)

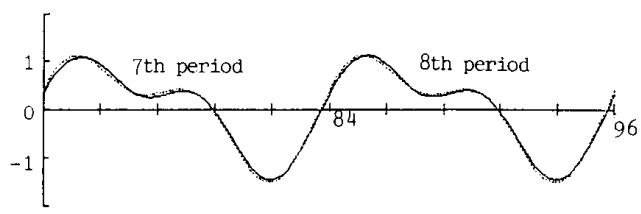

(c)

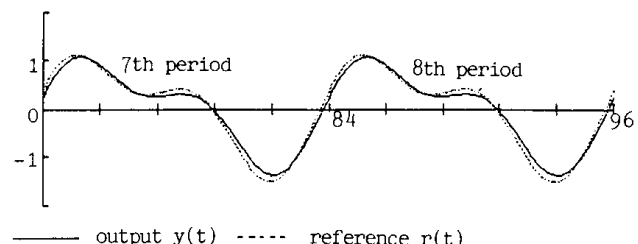

Fig. 12. Responses for the example: (a) $q(s)=0$, (b) $\rho=10^{5}, T=0.56$, (c) $\rho=10^{5}, T=1.0$

The last equation leads to

$$
\begin{aligned}
(I+G)^{-1} & =\left\{I+\left[I-N\left(X+K_{1}\right)\right]^{-1} N\left(X+K_{1}\right)\right\}^{-1} \\
& =I-N X-N K_{1}=\tilde{Y} \bar{D}-N K_{1}
\end{aligned}
$$

and then condition 2 ) in Corollary 1 is reduced to the existence of $K_{1}(s) \in \boldsymbol{R}_{-}^{m \times p}$ such that

$$
\left\|q\left(\tilde{Y} \tilde{D}-N K_{1}\right)\right\|_{\infty}<1 .
$$

Note that this condition does not depend on the free parameter $K_{2}(s)$, but only on $K_{1}(s)$. It follows from the relation $N X+\tilde{Y} \tilde{D}$ $=I$ that (4.18) can be rewritten as $\left\|q\left[I-N\left(X+K_{1}\right)\right]\right\|_{\infty}<1$, and the condition is reduced to $\|q\|_{\infty}<1$ by letting $K_{1}=-X$. Consequently, we see that the system can be stabilized by using a low-pass filter $q(s)$ near 1 . In other words, a modified repetitive control system with arbitrarily small steady-state error can be constructed. Note that this is not true in the single freedom case $C_{2}(s)=0$. It is readily seen from $(4.15 \mathrm{~b})$ that $C_{2}(s)=0$ is achieved by setting $K_{1}=K_{2} \tilde{D}$ and the stability condition is expressed as $\left\|q\left[\tilde{Y}-N K_{2}\right] \tilde{D}\right\|_{\infty}<1$. Therefore, $q(s)$ cannot in general tend to 1 in this case. However, noting that if the plant is stable, then we can set $\tilde{D}=\tilde{Y}=I$, the stability condition can be rewritten as $\left\|q\left[I-N K_{2}\right]\right\|_{\infty}<1$. If we set $K_{2}$ near zero, a similar discussion of the two-degree-of-freedom case concludes that the steady-state error can be arbitrarily small only by the cascade compensator $C_{1}(s)$ for stable plants.

We now clarify the class of $q(s)$ satisfying (4.4) or (4.18) under the following assumptions:

a) $q(j \omega) \neq 0 ; \forall \omega,|q(\infty)|<1$,

b) $\operatorname{rank}[P(j \omega)]=p ; \forall \omega$.

Using the inner-outer factorizations $q=q_{i} q_{0}$ ( $q_{i}$ is inner and $q_{o}$ is outer) and $N=N_{i} N_{o}$ ( $N_{i}$ is square inner and $N_{o}$ is outer), and recalling that $N_{i}^{\sharp}(s) N_{i}(s)=I$, where $N_{i}^{\sharp}(s) \triangleq N_{i}^{T}(-s)$ is also square inner, condition (4.18) can be rewritten as

$$
\begin{aligned}
& \left\|q_{i} q_{o}\left[\tilde{Y} \tilde{D}-N_{i} N_{o} K_{1}\right]\right\|_{\infty} \\
& \quad=\left\|q_{o} N_{i}^{\#}\left[\tilde{Y} \tilde{D}-N_{i} N_{o} K_{1}\right]\right\|_{\infty} \\
& \quad=\left\|q_{o} N_{i}^{\#} \tilde{Y} \tilde{D}-S_{1}\right\|_{\infty}<1
\end{aligned}
$$

where

$$
S_{1}(s)=q_{o}(s) N_{o}(s) K_{1}(s) \in \boldsymbol{R}_{-} .
$$

Since $N_{0}$ is an outer matrix, there exists a stable $m \times p$ matrix $N_{o}^{+}$such that $N_{o} N_{o}^{+}=I$. Hence, it is easily seen by setting $K_{1}=$ $q_{0}^{-1} N_{o}^{+} S_{1}$, which is stable but improper in general, that there exists a $K_{1}(s)$ satisfying (4.18) if $S_{1}(s)$ satisfies (4.20). It is well known that if we let $K_{1}^{\prime}=K_{1} /(1+\alpha s)^{l}$ for $K_{1}$ satisfying (4.18), sufficiently small positive number $\alpha$ for sufficiently large integer $l$ then $K_{1}^{\prime}$ is an $\boldsymbol{R}_{-}$matrix that satisfies (4.18) under the assumptions (4.19a) and (4.19b) [21]. Hence, the existence

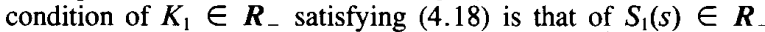
satisfying (4.20).

A similar discussion can be carried out for the single freedom case. We now impose an additional assumption on the plant

c) $P(s)$ has no pole on the imaginary axis.

In this case, there exists a co-inner-outer factorization $\tilde{D}=$ $\tilde{D}_{c o} \tilde{D}_{c i}\left(\tilde{D}_{c o}\right.$ is square co-outer with $\tilde{D}_{c o}^{-1} \in \boldsymbol{R}_{-}$and $\tilde{D}_{c i}$ is square co-inner) and the stability condition

$$
\left\|q\left[\tilde{Y}-N K_{2}\right] \tilde{D}\right\|_{\infty}<1 ; K_{2} \in \boldsymbol{R}_{-}
$$

reduces to

$$
\left\|q_{o} N_{i}^{\#} \tilde{Y} D_{c o}-S_{2}\right\|_{\infty}<1
$$

where

$$
S_{2}(s)=q_{o}(s) N_{o}(s) K_{2}(s) \tilde{D}_{c o}(s) \in \boldsymbol{R}_{-} .
$$

The derived stabilizability conditions $(4.20)$ and (4.23) for twodegree-of-freedom and one-degree-of-freedom cases are a kind of $H^{\infty}$ optimization problems. Thus, we can apply the results of the Hankel norm approximation [14] and the Nevanlinna-Pick interpolation [22], [13] and other techniques to solve the problem. An 
application of Nevanlinna-Pick interpolation can be found in [19] We will now apply the Hankel norm approximation technique. Define

$$
\begin{aligned}
& \Gamma_{1} \triangleq q_{o} N_{i}^{\sharp} \tilde{Y} \tilde{D}=\Gamma_{1 s}+\Gamma_{1 u}, \\
& \Gamma_{2} \triangleq q_{o} N_{i}^{\sharp} \tilde{Y} \tilde{D}_{c o}=\Gamma_{2 s}+\Gamma_{2 u},
\end{aligned}
$$

where $\Gamma_{i s}$ and $\Gamma_{i u}$ are the stable and unstable parts of $\Gamma_{i}$, respectively, for $i=1,2$; i.e., $\Gamma_{i s}(s)$ and $\Gamma_{i u}(-s)$ belong to $\boldsymbol{R}_{-}$. Since

$$
\inf _{S_{i} \in R_{-}}\left\|\Gamma_{i s}+\Gamma_{i u}-S_{i}\right\|_{\infty}=\inf _{S_{i} \in R_{-}}\left\|\Gamma_{i u}-\hat{S}_{i}\right\|_{\infty}=\left\|\Gamma_{i u}\right\|_{H} ; \quad i=1,2
$$

where $\|\cdot\|_{H}$ denotes the Hankel norm of the transfer function [14], we have the following stabilizability condition.

Theorem 3: Under the assumptions (4.19a) and $(4.19 \mathrm{~b})$, there exist $C_{i}(s) \in \boldsymbol{R}_{p}^{m \times p} ; i=1,2$ that satisfy conditions 1) and 2) in Corollary 1 , if and only if

$$
\left\|\Gamma_{1 u}\right\|_{H}<1
$$

where $\Gamma_{1 u}$ is the unstable part of $\Gamma_{1}=q_{o} N^{\#} \tilde{Y} \tilde{D}$.

Under the assumptions of $(4.19 \mathrm{a}),(4.19 \mathrm{~b})$, and $(4.19 \mathrm{c})$ and $C_{2}(s)=0$, there exists $C_{1}(s) \in \boldsymbol{R}_{p}^{m \times p}$ that satisfy conditions 1$)$ and 2 ) in Corollary 1 , if and only if

$$
\left\|\Gamma_{2 u}\right\|_{H}<1
$$

where $\Gamma_{2 u}$ is the unstable part of $\Gamma_{2}=q_{o} N_{i}^{\sharp} \tilde{Y} \tilde{D}_{c o}$.

Remark 1: Clearly, the unstable zeros of the plant (i.e., the zeros of $\left.\phi(s) \triangleq \operatorname{det}\left[N_{i}(s)\right]\right)$ are the unstable poles of $N_{i}^{*}$. Hence, by partial fraction expansion, we see that $\Gamma_{1 u}$ and $\Gamma_{2 u}$ depend on the value of $q\left(\lambda_{j}\right), j=1_{\sim} \mu$, where $\lambda_{j}, j=1_{\sim} \mu$ are the zeros of $\phi(s)$ (see Example 2 below). Consequently, Theorem 3 implies that there should be some kind of restriction on the values of $q\left(\lambda_{j}\right)$ in order to satisfy the stability condition and that we have the restriction of the frequency band to be tracked for nonminimum phase plants. This also means that any low-pass filter $q(s)$ can be selected for a minimum-phase plant, which has been pointed out in Section IV-A.

Remark 2: When $q(s)$ satisfies the condition of Theorem 3, $S_{1}(s)$ [respectively, $S_{2}(s)$ ] satisfying (4.20) [respectively, (4.23)] can be characterized using an appropriate strictly bounded real matrix $Z(s) \in R_{-}$, i.e., $\|Z\|_{\infty}<1$ [23]. Therefore, the class of stabilizing controllers is completely parametrized by $Z(s)$. The parametrization can also be obtained via the Nevanlinna-Pick theory [19].

Summarizing the above, the following synthesis algorithm is obtained for modified repetitive control systems.

[A Synthesis Algorithm by the Factorization Approach]:

Step 1: Determine the frequency band to be tracked, or the time constant $T$ in $q(s)=1 /(1+T s)$, satisfying the stabilizability condition in Theorem 3.

Step 2: Find the class of $S_{1}(s)$ [respectively, $S_{2}(s)$ ] satisfying (4.20) [respectively, (4.23)], which is parametrized by a strictly bounded real matrix and choose an appropriate free parameter $Z(s)$.

Step 3: Calculate the controller $C(s)$ by (4.15) with $K_{1}(s)=$ $N_{o}^{+}(S) S_{1}(s) /\left\{q_{o}(s)(1+\alpha s)\right\} \in \boldsymbol{R}_{-}$and an appropriate $\boldsymbol{R}$ matrix $K_{2}(s)$ [respectively, $K_{2}(s)=N_{a}^{+}(s) S_{2}(s) \tilde{D}_{c o}{ }^{-1}(s) /$ $\left\{q_{o}(s)(1+\alpha s)^{\prime}\right\} \in \boldsymbol{R}_{-}$and $\left.K_{1}(s)=K_{2}(s) D(s)\right]$, where $\alpha$ is a sufficiently small number and $l$ is a sufficiently large integer so that $K_{i}(s)$ is proper, $i=1,2$.

Example 2: We consider the stabilizability condition for an
SISO system described by $P(s)=(s-1) /(s+1)(s-2)$. Since

$$
\begin{gathered}
N=\tilde{N}=(s-1) /(s+1)^{2}, D=\tilde{D}=(s-2) /(s+1), \\
X=\tilde{X}=9, \quad Y=\tilde{Y}=(s-5) /(s+1)
\end{gathered}
$$

we have $N_{i}=\phi=(s-1) /(s+1), N_{i}^{\#}=(s+1) /(s-1)$, $\tilde{D_{c o}}=(s+2) /(s+1), \mu=1$, and $\lambda_{1}=1$. From these values we obtain

$$
\begin{aligned}
& \Gamma_{1}=q_{o}(s) \frac{(s-2)(s-5)}{(s-1)(s+1)}=\frac{-9 q_{o}(-1)}{s+1}+\frac{2 q_{o}(1)}{s-1}, \\
& \Gamma_{2}=q_{o}(s) \frac{(s-5)(s+2)}{(s-1)(s+1)}=\frac{3 q_{o}(-1)}{s+1}+\frac{-6 q_{o}(1)}{s-1} .
\end{aligned}
$$

Since $\|\beta /(s-\alpha)\|_{H}=|\beta / 2 \alpha| ; \alpha>0$, we have

$$
\begin{gathered}
\left\|\Gamma_{1 u}\right\|_{H}=\left\|\frac{2 q_{o}(1)}{s-1}\right\|_{H}=\left|q_{o}(1)\right|, \\
\left\|\Gamma_{2 u}\right\|_{H}=\left\|\frac{6 q_{o}(1)}{s-1}\right\|_{H}=3\left|q_{o}(1)\right| .
\end{gathered}
$$

Hence, the stabilizability condition is expressed as $\left|q_{o}(1)\right|<$ 1(respectively, $\left.\left|q_{0}(1)\right|<1 / 3\right)$ for two (respectively, one)degree-of-freedom case. For example, in the case $q(s)=1 /(1+$ $T S$ ), $T>0$ (respectively, $T>2$ ) is required for the stabilizability. Let $T$ be 3 , i.e., $q_{o}(1)=1 / 4$. Then the class of $S_{2}(s)$ satisfying (4.23) is represented by

$$
S_{2}(s)=[(s-1) z+3(s+1) / 4] /[3(s-1) z / 4+(s+1)]
$$

where $z(s) \in \boldsymbol{R}_{-}$and $\|z\|_{\infty}<1$. This parametrizes the class of stabilizing controllers with one-degree-of-freedom.

\section{CONCLUSION}

A new control scheme named repetitive control has been proposed. We have derived sufficient conditions for the stability of repetitive and modified repetitive control systems by applying the small gain theorem and the stability theorem for time-lag systems. Synthesis algorithms are presented both by the statespace approach and the factorization approach, and the class of stabilizing controllers and the low-pass filters has been characterized using the technique of the Hankel norm approximation.

The scheme can also be applied to a class of nonlinear systems such as multilink manipulators. The repetitive operation for the trajectory control can reduce the tracking error to a lower level [5], [26]. Furthermore, it is also useful for periodic disturbance inputs. The application to the attenuation of rotational fluctuations synthesized with the motor speed has been shown in [24].

An interesting topic for future study is to investigate the robust stability or stabilizability and the optimal design problem with an appropriate performance index.

\section{APPENDIX A}

Proof of Proposition 2

For simplicity, we prove the fact for the SISO case; more detailed analysis may be found in [25]. We employ the notation in the proof of Theorem 1. As shown there, $\lambda=C$ belongs to the spectrum $\sigma(\boldsymbol{A})$ if and only if

$$
\begin{aligned}
& \operatorname{det}(\lambda I-A) \operatorname{det}\left[(1-D \sigma)-C(\lambda I-A)^{-1} B \sigma\right]=0 \\
&(\sigma=\exp (-\lambda L)) .
\end{aligned}
$$

Now as shown in Hale [11, Lemma 1.7.1], there exists a sequence $\left\{\lambda_{j}\right\} \subset \sigma(A)$ such that $\operatorname{Re} \lambda_{j} \rightarrow \log D$. Since $G(s)$ is strictly 


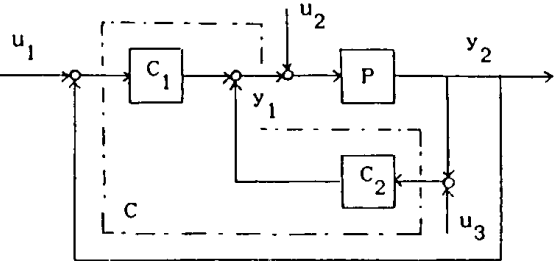

Fig. 13. A control system with two-degree-of-freedom controller.

proper, $D$ must be one because it is the constant term of $(1+$ $a G)^{-1}(1+(a-1) G)$. Therefore, the least upper bound of $\{\operatorname{Re}$ $\lambda ; \lambda \in \sigma(A)\}$ is no less than zero. Hence, again by Hale [11, Corollary 1.7 .1$]$, the closed-loop system cannot be exponentially stable.

\section{APPENDIX B}

Derivation of the Class of Stabilizing Controllers (4.15a) AND $(4.15 \mathrm{~b})$

Since $(I+G)^{-1} G$ in $\boldsymbol{R}_{-}$implies $(I+G)^{-1}$ belongs to $\boldsymbol{R}_{-}$, condition 1) in Corollary 1 is equivalent to the input-output stability of a system with a two-parameter compensator shown in Fig. 13, i.e., the transfer matrix from $\left(u_{1}, u_{2}, u_{3}\right)$ to $\left(y_{1}, y_{2}\right)$ belongs to $\boldsymbol{R}_{-}$. The configuration of Fig. 13 is similar to but slightly different from the one investigated by Vidyasagar [13, sect. 5.6]. If we replace $C_{2}$ by $C_{1}+C_{2}$ in [13], we obtain Fig. 13 . Hence, by using (5.6.16) in [13] the stabilizing controllers are parametrized as follows:

$$
\begin{gathered}
C_{1}=\left(Y-R_{2} \tilde{N}\right)^{-1} R_{1}, \\
C_{1}+C_{2}=\left(Y-R_{2} \tilde{N}\right)^{-1}\left(X+R_{2} \tilde{D}\right),
\end{gathered}
$$

i.e.,

$$
C_{2}=\left(Y-R_{2} \tilde{N}\right)^{-1}\left(X-R_{1}+R_{2} \tilde{D}\right) .
$$

Set $R_{1}$ and $R_{2}$ be $K_{1}+X$ and $K_{2}$, respectively, in (B.1) and (B.3), where $K_{1}$ and $K_{2}$ are in $\boldsymbol{R}_{-}$, then we have the parametrization (4.15).

\section{ACKNOWLEDGMENT}

The authors would like to thank Prof. M. Ikeda for his helpful discussions concerning with the stability conditions. They also wish to extend their gratitude to one of the referees whose comments greatly improved the final manuscript.

\section{REFERENCES}

[1] B. A. Francis and W. M. Wonham, "The internal model principle for linear multivariable regulators," Appl. Math. Opt., vol. 2, pp. 170$194,1975$.

[2] T. Inoue, M. Nakano, and S. Iwai, "High accuracy control of servomechanism for repeated contouring," in Proc. 10th Annual Symp. Incremental Motion Contr. Syst. and Devices, 1981, pp. Symp. Im
$258-292$

[3] T. Inoue et al., "High accuracy control of a proton synchrotron magnet power supply," in Proc. 8th World Congress of IFAC, 1981, vol. $\mathrm{xx}$, pp. 216-221.

[4] N. Nakano and S. Hara, "Microprocessor-based repetitive control," in Microprocessor-Based Control Systems. Amsterdam, The Netherlands, Reidel, 1986.

[5] S. Hara, T. Omata, and M. Nakano, "Synthesis of repetitive control systems and its application," in Proc. 24th Conf. Decision Contr., 1985, pp. 1384-1392.

[6] M. Uchiyama, "Formation of high-speed motion pattern of a mechanical arm by trail" (in Japanese), Trans. SICE, vol. 14, pp. 706-712, 1978.

[7] S. Arimoto, S. Kawamura, and F. Miyazaki, "Bettering operation of dynamic systems by learning: A new control theory for servo mechanism or mechatronics systems," in Proc. 23rd Conf. Decision Contr., 1984, pp. 1064-1069.
[8] S. Arimoto, S. Kawamura, and F. Miyazaki, "Bettering operation of robots by learning," J. Robotic Syst., vol. 1, pp. 123-140, 1985.

[9] S. Arimoto, S. Kawamura, F. Miyazaki, and S. Tamaki, "Learning control theory for dynamical systems," in Proc. 24th Conf. Decision Contr., 1985, pp. 1375-1380.

[10] T. Mita and E. Kato, "Iterative control and its application to motion control of robot arm-A direct approach to servo problems," in Proc. 24th Conf. Decision Contr., 1985, pp. 1393-1398.

[11] J. Hale, Theory of Functional Differential Equations. New York: Springer-Verlag, 1977.

[12] H. Kimura, "A new approach to the perfect regulation and bounded peaking in linear multivariable control systems," IEEE Trans. Automat. Contr., vol. AC-26, pp. 253-270, 1981.

[13] M. Vidyasagar, Control System Synthesis: A Factorization Approach. Cambridge, MA: M.I.T. Press, 1985

[14] K. Glover, "All optimal Hankel-norm approximations of linear multivariable systems and their $L^{\infty}$-error bounds," Int. J. Contr., vol. 39, pp. 1115-1193, 1984.

[15] C. A. Desoer and M. Vidyasagar, Feedback Systems: Input-Output Properties. New York: Academic, 1975.

[16] V. M. Kolmanovskii and V. R. Nosov, Stability of Functional Differential Equations. New York: Academic, 1986.

[17] W. B. Castelan, "A Lyapunov functional of a matrix neutral difference-differential equation with one delay," J. Math. Anal. Appl., vol. 71, pp. 105-130, 1979.

[18] D. Salamon, Control and Observation of Neutral Systems. New York: Pitman, 1984

[19] S. Hara and Y. Yamamoto, "Stability of repetitive control systems," in Proc. 24th Conf. Decision Contr., 1985, pp. 326-327.

[20] S. Hara, "Parameterization of stabilizing controllers for multivariable servo systems with two degrees of freedom," Int. J. Contr., vol. 45, pp. $779-790,1987$

[21] B. A. Francis, J. W. Helton, and G. Zames, " $H^{\infty}$-optimal feedback controllers for linear multivariable systems," IEEE Trans. Automat. Contr., vol. AC-29, pp. 888-900, 1984.

[22] P. H. Delsarte, Y. Genin, and Y. Kamp, "The Nevanlinna-Pick problem for matrix-valued functions," SIAM J. Appl. Math., vol. 36 , pp. 47-61, 1979 .

[23] K. Glover, "Robust stabilization of linear multivariable systems: Relation to approximation," Int. J. Contr., vol. 43, pp. 741-766, 1986.

[24] F. Kobayashi, S. Hara, H. Tanaka, and M. Nakano, "Reduction of rotational speed fluctuation in motors using the repetitive control" (in Japanese), Trans. IEE Japan, vol. 107-D, pp. 29-34, 1987.

[25] Y. Yamamoto and S. Hara, "Relationships between internal and external stability for infinite-dimensional systems with applications to a servo problem." in Proc 26th Conf Decision Contr. 1987 pp. 1558-1563; also IEEE Trans. Automat. Contr., submitted for publication.

[26] T. Omata, S. Hara, and M. Nakano, "Nonlinear repetitive control with application to trajectory control of manipulators," J. Robotic Syst., vol. 4, pp. 631-652, 1987 .

Shinji Hara (M'87), for a photograph and biography, see p. 67 of the January 1988 issue of this TRANSACTIONS.

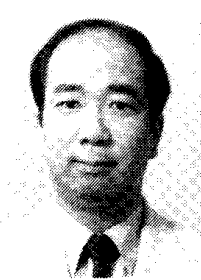

Yutaka Yamamoto (M'83) received the B.S. and M.S. degrees in engineering from Kyoto University, Kyoto, Japan, in 1972 and 1974, respectively, and the M.S. and Ph.D. degrees in mathematics from the University of Florida, in 1976 and 1978 , respectively.

From 1978 to 1987 he served as a Research Associate in the Department of Applied Mathematics and Physics, Kyoto University. In 1987 he joined the Department of Applied Systems Science as an Associate Professor. His current research interests are in realization theory and approximation of distributed parameter systems and infinite-dimensional servo systems. 
Dr. Yamamoto is a member of the Society of Instrumentation and Control Engineers and the Japan Association of Automatic Control Engineers.

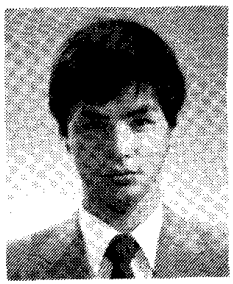

Tohru Omata was born in Tokyo, Japan, in 1959 He received the B.E., M.E., and Ph.D. degrees from Tokyo Institute of Technology, Tokyo, Japan, in 1981,1983 , and 1986 , respectively.

$\mathrm{He}$ is currently a Researcher in the Electrotechnical Laboratory, Tsukuba, Japan. His current research interests are in robotics, control theory, and artificial intelligence.

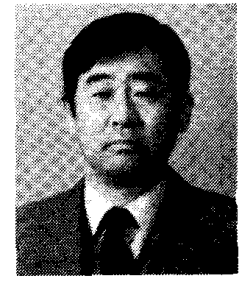

Michio Nakano (M'79) was born in Ibaragi, Japan, on February 17, 1939. He received the B.S., M.S., and $P h . D$. degrees in electrical engineering from the Tokyo Institute of Technology, Tokyo, Japan, in 1963,1965 , and 1968 , respectively.

Since 1968, he has been with the Department of Control Engineering, Tokyo Institute of Technology, where he is currently a Professor. He has also been a Visiting Professor at the National Laboratory for High-Energy Physics, Tsukuba, Japan, since 1975. His research interests are in motion control systems and devices and in the applications of control theory to electrical systems.

Dr. Nakano is a member of the IEE of Japan and the Society of Instrument and Control Engineers of Japan. 